\title{
Effects of peanut meal extracts fermented by Bacillus natto on the growth performance, learning and memory skills and gut microbiota modulation in mice
}

\author{
Xiaoyang Jiang ${ }^{1,2} \dagger$, Haoyue Ding ${ }^{1} \dagger$, Qing Liu $^{1}$, Yuxi Wei ${ }^{2}$, Yuanjie Zhang ${ }^{3}$, Yanping Wang ${ }^{4}$, Yaqian Lu $^{1}$, \\ Aiguo $\mathrm{Ma}^{1}$, Zichao $\mathrm{Li}^{2} *$ and Yingfen $\mathrm{Hu}^{2 *}$ \\ ${ }^{1}$ School of Public Health, Medical College, Qingdao University, Qingdao 266071, Shandong, People's Republic of China \\ ${ }^{2}$ College of Life Sciences, Institute of Advanced Cross-Field Science, Qingdao University, Qingdao 266071, Shandong, People's \\ Republic of China \\ ${ }^{3}$ Health Supervision Institute, Xuzhou 221000, Jiangsu, People's Republic of China \\ ${ }^{4}$ Center for Disease Control and Prevention, Feixian County 273400, Shandong, People's Republic of China
}

(Submitted 23 April 2019 - Final revision received 26 October 2019 - Accepted 13 November 2019 - First published online 6 November 2019)

\section{Abstract}

Recent studies have demonstrated that the nutritional properties of peanut meal (PM) can be improved after being fermented. The assessment of fermented PM has been reported to be limited to various physical and chemical evaluations in vitro. In the present study, PM was fermented by Bacillus natto to explore the effects of fermented PM extract (FE) on growth performance, learning and memory ability and intestinal microflora in mice. Ninety newly weaned male Kunming (KM) mice were randomly divided into seven groups: normal group ( $n 20)$, low-dose FE group $(n$ 10), middle-dose FE group (MFE) ( $n$ 10), high-dose FE group (HFE) ( $n$ 20), unfermented extraction group ( $n$ 10), model group (10) and natural recovery group (10). Learning and memory skills were performed by the Morris water maze (MWM) test, and the variation in gut microbiota (GM) composition was assessed by $16 \mathrm{~S}$ rDNA amplicon sequencing. The results show that HFE remarkably improved the growth performance in mice. In the MWM test, escape latency was shortened in both MFE and HFE groups, while the percentage of time, distance in target quadrant and the number crossing over the platform were significantly increased in the HFE group. Moreover, the FE played a preventive role in the dysbacteriosis of mice induced by antibiotic and increased the richness and species evenness of GM in mice.

Key words: Bacillus natto: Peanut meal: Growth performance: Learning: Memory: Gut microbiota

Bacillus natto is a subspecies of Bacillus subtilis. It belongs to the Gram-positive aerobic bacteria species with long rod-like cells, acid and heat resistance and strong pathogenic bacteria inhibition ability ${ }^{(1,2)}$. B. natto can secrete a variety of proteases (especially alkaline protease), glucoamylase, lipase and amylase. It can decompose proteins, fats, carbohydrates and other macromolecular substances into small peptides, amino acids, organic acids, oligosaccharides and other components that are easily absorbed by the human body and can produce amino acids, thereby improving the utilisation of fermentation products $^{(3,4)} . B$. natto has been reported to show a variety of functions, including blood pressure lowering ${ }^{(5)}$, anti-tumour ${ }^{(6)}$, thrombolytic ${ }^{(7)}$, etc. Moreover, as a probiotic, it plays an effective role in animals through the following mechanisms: production of antibiotics, competition with harmful bacteria for nutrition, alleviation of emergency response and enhancement of immunity ${ }^{(8)}$. Therefore, $B$. natto has become a research hotspot these years. As one of the forty probiotics announced by the US Food and Drug Administration, it has been widely used in medicine, health food, agriculture, animal husbandry and other fields to date ${ }^{(9)}$.

Infant period is the fastest growing period after birth, in which adequate nutrition can lay a good foundation for health throughout life. It is not only necessary for physical growth of infants and young children but also the material basis for the formation of social behavioural abilities such as cognitive ability, language development and intelligence, physical strength and immunity ${ }^{(10)}$. And among which protein, sugar and flavonoids are important

Abbreviations: FE, fermented peanut meal extract; GM, gut microbiota; HFE, high-dose FE group; MFE, middle-dose FE group; MWM, Morris water maze; OTU, operational taxonomic unit; PM, peanut meal; UFE, unfermented extraction group.

* Corresponding authors: Yingfen Hu, email qingdahyf2006@163.com; Zichao Li, email zichaoli@qdu.edu.cn

$\dagger$ These authors contributed equally to this work. 
factors that can affect brain development and growth. The fermentation products of $B$. natto have been reported to exhibit high antioxidant activity, and good scavenging capacity of 2,2'diphenyl-1-pyridine hydrazine free radicals and hydroxyl free radicals $^{(11,12)}$ which is so important for eliminating excessive free radicals in the body and improving or enhancing cognitive ability ${ }^{(13-15)}$. In addition, antioxidants have been found to improve learning ability. Extracts of natural products such as onion ${ }^{(16)}$ and green tea ${ }^{(17)}$ can reverse memory impairment, reduce lipid peroxides and increase antioxidant enzyme activity in brain. The antioxidant capacity for fermentation broth of B. subtilis has important research significance for improving current human growth with age, cognitive decline and children's growth and development ${ }^{(18,19)}$.

The regulation of gut microbiota (GM) has become an important way for humans to treat gastrointestinal and metabolic diseases $^{(20)}$, and it plays an important role in the intestinal morphology, immunity, digestion and health of the host ${ }^{(21,22)}$. A healthy GM ecology is critical for the establishment of tissue homeostasis health. Gut homeostasis plays a crucial role in maintaining animal and human health and is depended on the regulation of the microbiota ${ }^{(23)}$. Therefore, regulating the richness or diversity of intestinal microbes, thereby promoting the normal metabolism of GM, is of great significance to the health of the human body. Studies have shown the beneficial effects of probiotics in improving the GM, and they can further promote human health, immunity and digestion ${ }^{(24,25)}$. It has been reported that $B$. subtilis can effectively regulate $\mathrm{GM}^{(26,27)}$. It was demonstrated that a higher dose of $B$. subtilis administration ameliorated dextrose sulfate sodium (DSS)-induced dysbiosis and gut inflammation by balancing beneficial and harmful bacteria and associated anti- and pro-inflammatory agents, therefore aiding intestinal mucosa recovery from DSS-induced injuries ${ }^{(28)}$. It was also well documented that $B$. subtilis can improve $\mathrm{GM}^{(29)}$. As one of B. subtilis, B. natto, showing remarkable potential in regulating $\mathrm{GM}$, has important clinical significance for lipid lowering and anti-cancer.

Peanut meal (PM) is a by-product of peanut oil pressed at high temperature ${ }^{(30)}$. It is rich in protein, peanut fibre, minerals, vitamins, peanut polyphenols, etc. The protein content is as high as $47-55 \%$, and the biotin content is $1.79 \mathrm{mg} / \mathrm{kg}^{(31)}$. China is the world's largest producer for peanuts, of which about 50-60\% are used to extract oil, producing millions of tons of petroleum by-products (PM) per year ${ }^{(32,33)}$. However, PM has been merely utilised as an animal feed or even fertiliser so far, due to its protein denaturation caused by high-temperature pressing, imbalance of amino acid content and high-level aflatoxin contamination $^{(34,35)}$, causing waste issue to these mass resources. Herein, the high-value utilisation of PM remains challenging to date ${ }^{(29)}$. Recently, evidences suggest that the microbial fermentation of PM can significantly improve its nutritional characteristics, increase the content of protein, small molecular peptide, amino acid and organic acid and improve its digestibility in vitro, which provides a new approach to settle the matter ${ }^{(32,36)}$. Nonetheless, rare in-depth studies can be found on the benefits of PM fermented by B. subtilis species in experiments in vivo so far.

In the present study, mice were fed with PM extract fermented by $B$. natto to study the regulation of growth performance, learning and memory ability and GM in mice, which provided important reference for high-value utilisation and clinical research of PM.

\section{Materials and methods}

\section{Animals}

A total of ninety weanling male Kunming (KM) mice (20 (SE 2) g) were purchased from the Experimental Animal Center of Shandong Lukang Pharmaceutical Co. Ltd (animal quality qualification certificate no. SCXK (Lu) 20140001). The mice were fed with sterile food and water, and the temperature in the animal room was maintained at $24(\mathrm{se} 2)^{\circ} \mathrm{C}$ with a $12 \mathrm{~h}$ light $-12 \mathrm{~h}$ dark cycle.

\section{Materials}

B. subtilis was isolated from Japanese natto, which was preserved in Food Engineering Laboratory in Qingdao University. The PM was supplied by Qingdao Jiali Group Co. Ltd. The soyabean meal and wheat bran were purchased from local market. The mainly used agents were as follows: ampicillin (Zhuhai federal pharmaceutical Co. Ltd); Phusion ${ }^{\circledR}$ High-Fidelity PCR Master Mix with GC Buffer (New England Biolabs); Gene JET Gel Extraction Kit (Thermo Scientific); IlluminaTruSeq DNA PCR-Free Library Preparation Kit (Illumina) and Qubit@ 2.0 Fluorometer (Thermo Scientific). And, other chemical reagents were purchased from Sinopharm Chemical Reagent Co. Ltd.

\section{Preparation of fermented extracts from peanut meal}

PM-soyabean meal-bran (7:2:1) was placed in a fermentation medium, and then the seed liquid of $B$. natto was inoculated. The mixture was cultured for $38 \mathrm{~h}$ at $37^{\circ} \mathrm{C}$ afterwards ${ }^{(37)}$. Ferments were centrifuged, filtered and freeze-dried for reservation (fermented PM extract (FE)).

\section{Composition determination in fermented peanut meal extract}

The compositions to be analysed in FE and the related test methods are listed in Table 1.

\section{Experimental groups}

Expt I: After a week of habituation, mice were randomly divided into five groups as follows: (N) normal group: saline; low-dose FE group: $0.3 \mathrm{~g} / \mathrm{kg}$ per d; middle-dose FE group (MFE): $1.5 \mathrm{~g} / \mathrm{kg}$

Table 1. Composition in fermented peanut meal extract and determination methods

\begin{tabular}{ll}
\hline $\begin{array}{l}\text { Composition } \\
\text { determination }\end{array}$ & Method \\
\hline Nattokinase activity & $\begin{array}{l}\text { Folin phenol method(38) } \\
\text { Acid hydrolysis method }\end{array}$ \\
Fat & Kjeldahl method for determination of $\mathrm{N}^{(40)}$ \\
Protein & Ninhydrin method $^{(41)}$ \\
Free amino acid & $\mathrm{Biuret}^{(42)}$ \\
Polypeptide & $\mathrm{NaNO}_{2}-\mathrm{Al}\left(\mathrm{NO}_{3}\right)_{3}-\mathrm{NaOH}$ chromogenic method \\
Total flavonoids & Phenol-sulphuric acid method $^{(44)}$ \\
Polysaccharide & Plate-coating method $^{(45)}$ \\
Live bacteria & \\
\hline
\end{tabular}


per d; high-dose FE group (HFE): $7.5 \mathrm{~g} / \mathrm{kg}$ per d and unfermented extraction group (UFE). Mice were gavaged once per $\mathrm{d}$ for 4 weeks, and the dosage for each gavage was $0.1 \mathrm{ml} / \mathrm{g}$ body weight $(\mathrm{BW})$ per $\mathrm{d}$. And then, ten mice from each group were randomly selected for the evaluation of growth performance, learning and memory skills.

Expt II: Regulation of PM fermentation on antibiotic-induced microbial imbalance in mice. After 1 week of adaptive feeding, mice were randomly divided into four groups: (A) normal group; (B) model group; (C) natural recovery group; (D) FE group. Except for normal saline group, the other groups were given FE for 4 weeks. The dosage of FE was the same as that of Expt I. After 4 weeks, except normal saline group, the other three groups began to induce diarrhoea: (B) model group: ampicillin; (C) natural recovery group: ampicillin + saline; (D) FE group: ampicillin + FE $(0.1 \mathrm{ml} / \mathrm{g}$ BW per d). Among them, ampicillin dose was $2 \mathrm{mg} / \mathrm{g} \mathrm{BW}$ per $\mathrm{d}$, which was five times the adult dose. The mice in each group were killed after 1 week of continuous intragastric administration. In an aseptic environment, the intestinal contents were taken out and frozen and stored at $-80^{\circ} \mathrm{C}$ for later evaluation.

\section{Morris water maze tests}

After intervention for 4 weeks, learning and memory skills were measured by the Morris water maze (MWM) tests ${ }^{(46)}$. The experiment was performed as previously described ${ }^{(47-49)}$ : Mice were trained for $5 \mathrm{~d}$. Daily training comprised of four sessions, starting at different quadrants of the water tank. The latency was recorded to reach the hidden platform. The rats were trained four times/d and put into the water from four entry points for $5 \mathrm{~d}$. The time from entering water to climbing the platform within $60 \mathrm{~s}$ was recorded, that is, escape latency. If the mice did not find the platform within $60 \mathrm{~s}$, it was induced to the platform with a rod and kept for $10 \mathrm{~s}$. The incubation period was recorded as $60 \mathrm{~s}$. After $5 \mathrm{~d}$, the platform was removed and the mice were placed in the opposite side of the original platform quadrant. Then, the time ratio (the ratio of swimming time:total swimming time in the target quadrant), the distance ratio (the ratio of swimming distance:total swimming distance in target quadrant) and the number crossing over the position of the platform were recorded. Lastly, the data were recorded by an MWM image analysis system (Mobile Datum). After 24 h of MWM test, mice were killed by dislocation. Liver, spleen and kidney were separated immediately. The mice were weighed on ice and frozen in liquid $\mathrm{N}_{2}$ and then preserved at $-80^{\circ} \mathrm{C}$ for later evaluation.

\section{5 rDNA amplicon sequencing}

Faecal bacterial DNA extraction. The DNA of faecal bacteria was extracted by cetyltrimethylammonium bromide/sodium dodecyl sulfate (SDS). The faecal DNA samples were concentrated and purified by $1 \%$ agarose gel electrophoresis and UV spectrophotometer. Then, it was diluted to $1 \mathrm{ng} / \mathrm{ul}$ with sterile water according to the concentration and was preserved at $-80^{\circ} \mathrm{C}$ as a template for $16 \mathrm{~S} \mathrm{rDNA}$ analysis.

16S rDNA gene PCR amplification and sequencing. The primers $515 \mathrm{~F}$ (5'-CACGGTCGKCGGCGCCATT-3') and $806 \mathrm{R}\left(5^{\prime}\right.$ GGACTACHVGGGTWTCTAAT- $\left.3^{\prime}\right)^{(50,51)}$ were used to amplify the V4 domain of bacterial 16S rDNA. And then, 5-10 ng DNA templates, $15 \mu$ l of Phusion ${ }^{\circledR}$ High-Fidelity PCR Master Mix and $2 \mu \mathrm{M}$ of each primer were contained in $30 \mu \mathrm{l}$ PCR reaction. Reaction conditions were as follows: an initial denaturation at $98^{\circ} \mathrm{C}$ for $1 \mathrm{~min}$, followed by thirty cycles of $98^{\circ} \mathrm{C}$ for $10 \mathrm{~s}, 50^{\circ} \mathrm{C}$ for $30 \mathrm{~s}$ and $72^{\circ} \mathrm{C}$ for $30 \mathrm{~s}$ and a final extension of $72^{\circ} \mathrm{C}$ for $5 \mathrm{~min}$. The same volume of $1 \times$ loading buffer (contained SYBR $^{\circledast}$ Green) was mixed with PCR products and went for electrophoresis detection on $2 \%$ agarose gel. The samples with bright main strip between 400 and 450 bp were chosen for further experiments. PCR products were mixed in equidensity ratios. Subsequently, mixture PCR products were purified with a Gene JET Gel Extraction Kit. Sequencing libraries were generated using an Illumina TruSeq DNA PCR-Free Library Preparation Kit following manufacturer's recommendations, and index codes were added. The library quality was assessed on Qubit@2.0 Fluorometer and Agilent Bioanalyzer 2100 system. Finally, the library was sequenced on an Illumina HiSe platform, and $250 \mathrm{bp}$ paired-end reads were generated.

165 rDNA gene analysis. Paired-end reads from the original DNA fragments were merged using FLASH $^{(52)}$, a very fast and accurate analysis tool which is designed to merge paired-end reads when there were overlaps between reads 1 and reads 2 . Sequences were analysed using QIIME software package (Quantitative Insights into Microbial Ecology) ${ }^{(53)}$, and in-house Perl scripts were used to analyse $\alpha$ - (within samples) and $\beta$ (among samples) diversity. Sequences with $\geq 97 \%$ similarity were assigned to the same operational taxonomic unit (OTU). A representative sequence for each OTU was picked, and the RDP classifier ${ }^{(54)}$ was used to annotate taxonomic information for each representative sequence. In order to compute $\alpha$ diversity, the OTU table was rarified and three metrics were calculated: Chao1 which estimates the species abundance; Observed Species which estimates the amount of unique OTU found in each sample and Shannon index. Rarefaction curves were generated based on the three metrics. Significant differences in taxonomic abundance between groups were tested by using the Wilcoxon rank sum test.

\section{Statistical analysis}

Data analyses were performed with SPSS Statistics 17.0 software. The significant differences of data were analysed by one-way ANOVA with Fisher's least-significant difference (LSD) test. All results are presented as mean values with their standard errors, and the significant criterion was set for $P<0 \cdot 05$.

\section{Results}

\section{Main composition of fermented peanut meal extract}

The tested results of the main composition of FE are listed in Table 2.

\section{Effects of growth and development}

The effects of FE on growth performance in mice were investigated, and the results are shown in Fig. 1. Compared with group N, 
Table 2. Determined main composition in fermented peanut meal extract

\begin{tabular}{lcccccccc}
\hline Composition & $\begin{array}{c}\text { Nattokinase } \\
(\mathrm{U} / \mathrm{g})\end{array}$ & $\begin{array}{c}\text { Fat } \\
(\%)\end{array}$ & $\begin{array}{c}\text { Protein } \\
(\mathrm{mg} / \mathrm{ml})\end{array}$ & $\begin{array}{c}\text { Free amino } \\
\text { acid }(\mathrm{mg} / \mathrm{ml})\end{array}$ & $\begin{array}{c}\text { Polypeptide } \\
(\mathrm{mg} / \mathrm{ml})\end{array}$ & $\begin{array}{c}\text { Polysaccharide } \\
(\mathrm{mg} / \mathrm{ml})\end{array}$ & $\begin{array}{c}\text { Total flavonoids } \\
(\mathrm{mg} / \mathrm{ml})\end{array}$ & $\begin{array}{c}\text { viable bacteria } \\
\text { (colony-forming units/ml) }\end{array}$ \\
\hline Content & 1360 & 2.35 & 70 & 54.89 & 10.32 & 10.36 & 10.28 & $4.2 \times 10^{16}$ \\
\hline
\end{tabular}

(A)

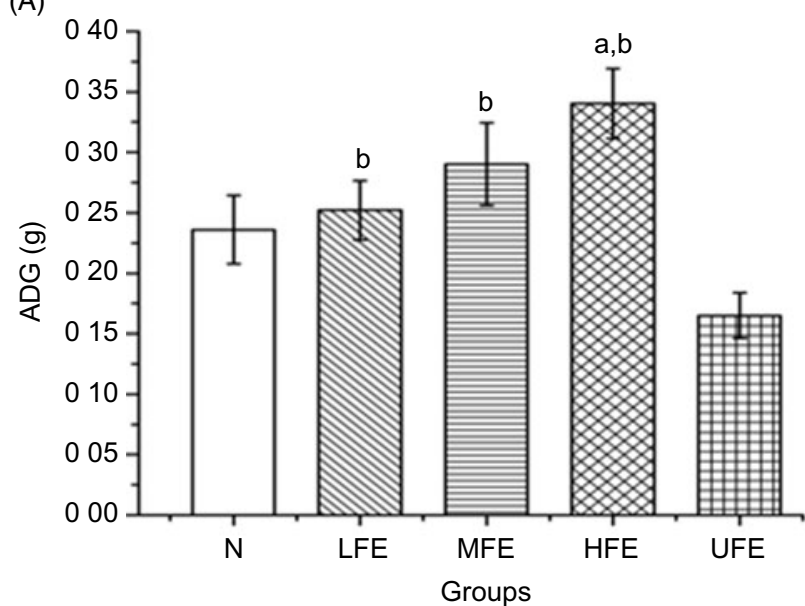

(C)

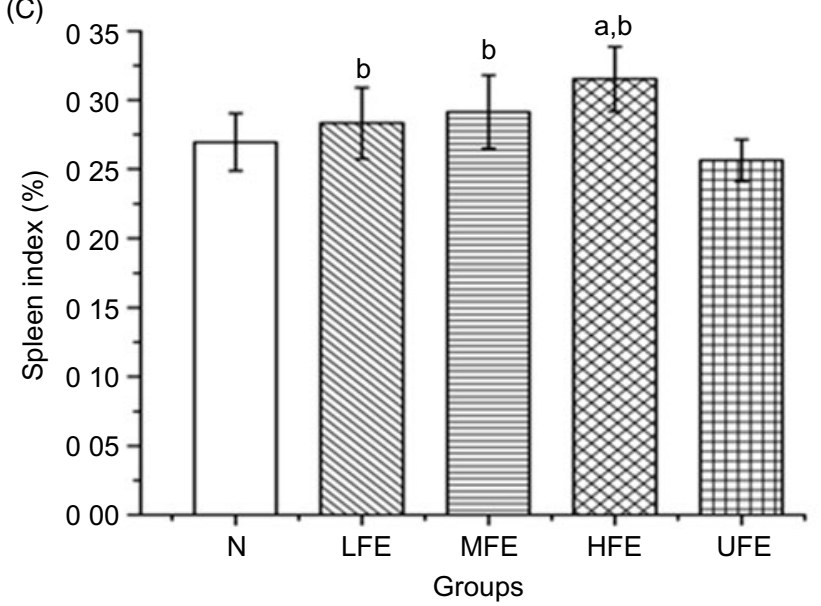

(B)

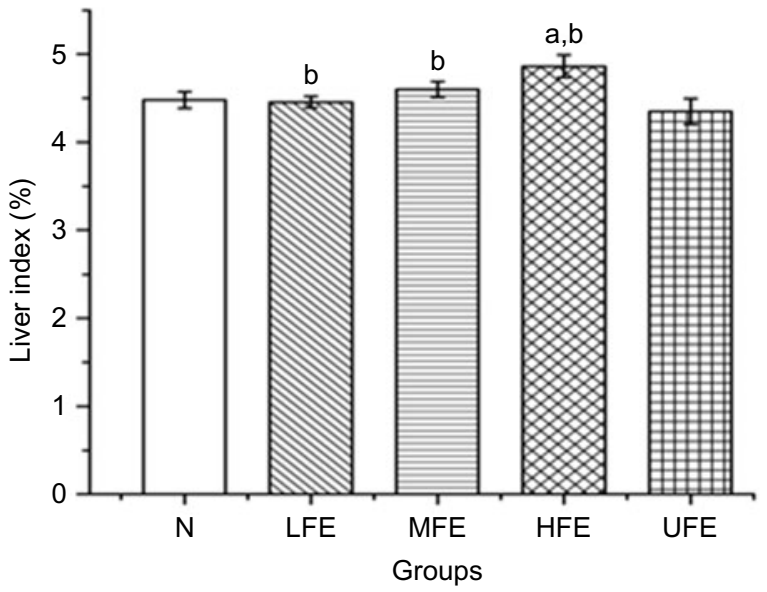

(D)

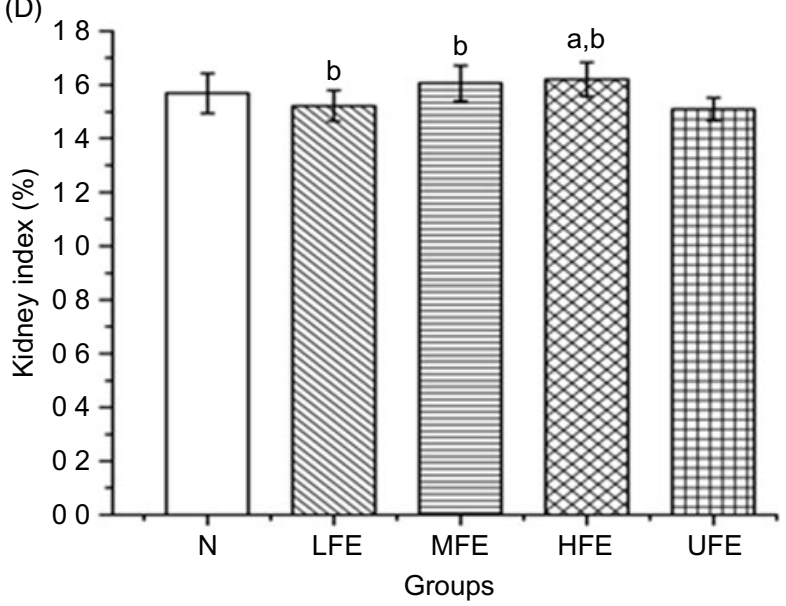

Fig. 1. (A) Average daily body weight gain (ADG) of mice in each group; (B) liver index of mice in each group; (C) spleen index of mice in each group; (D) kidney index of mice in each group. $A D G=($ last weight - first weight)/test date; viscera index $=$ viscera weight $(\mathrm{g}) /$ body weight $(\mathrm{g}) \times 100 \%$; values are presented as mean values with their standard errors. a $P<0.05 \mathrm{v}$. N group; ${ }^{\mathrm{b}} P<0.05 \mathrm{v}$. UFE group. N, normal group; LFE, low-dose of fermented peanut meal extract (FE) group; MFE, middle-dose of FE group; HFE, high-dose of FE group; UFE, unfermented extraction group.

average daily weight gain (ADG) index of mice in low-dose FE group, MFE and HFE was significantly increased and there were significant differences in the HFE group $(P<0.05)$, as shown in Fig. 1(A). It can be observed from Fig. 1(B)-(D) that there were no notable differences in liver, spleen and kidney index between low-dose FE group, MFE, HFE and group N $(P>0.05)$. The growth and organ index were affected by the fermentation products. It can be inferred from the effect of FE on growth and organ indexes of experimental mice that FE showed no harmful effect to the health of experimental animals.

\section{Morris water maze test}

The MWM was performed to evaluate the effects of FE on the learning and memory skills of mice. The swimming trajectory of each experimental group during probe tracking is shown in Fig. 2(A).

As shown in Fig. 2(B), the escape latencies during training tests showed remarkable differences within each group. On the 1st day of training, the average latency of mice in each group exhibited no statistical difference $(P>0 \cdot 05)$. On the 2 nd day, the average latency of mice in the HFE group was significantly shorter than that in N group and UFE group $(P<0.05)$. On the $3 \mathrm{rd}$ and 4 th days, the average latency of mice in MFE and HFE groups was significantly shorter than that in $\mathrm{N}$ and UFE groups $(P<0.05)$. And on the 5 th day, the average latency of mice in the HFE group was significantly shorter than that in N group and UFE group $(P<0.05)$.

After $5 \mathrm{~d}$ of training, the platform was removed and the probe trajectory was started. The results are shown in Fig. 2(C)-(F). The 

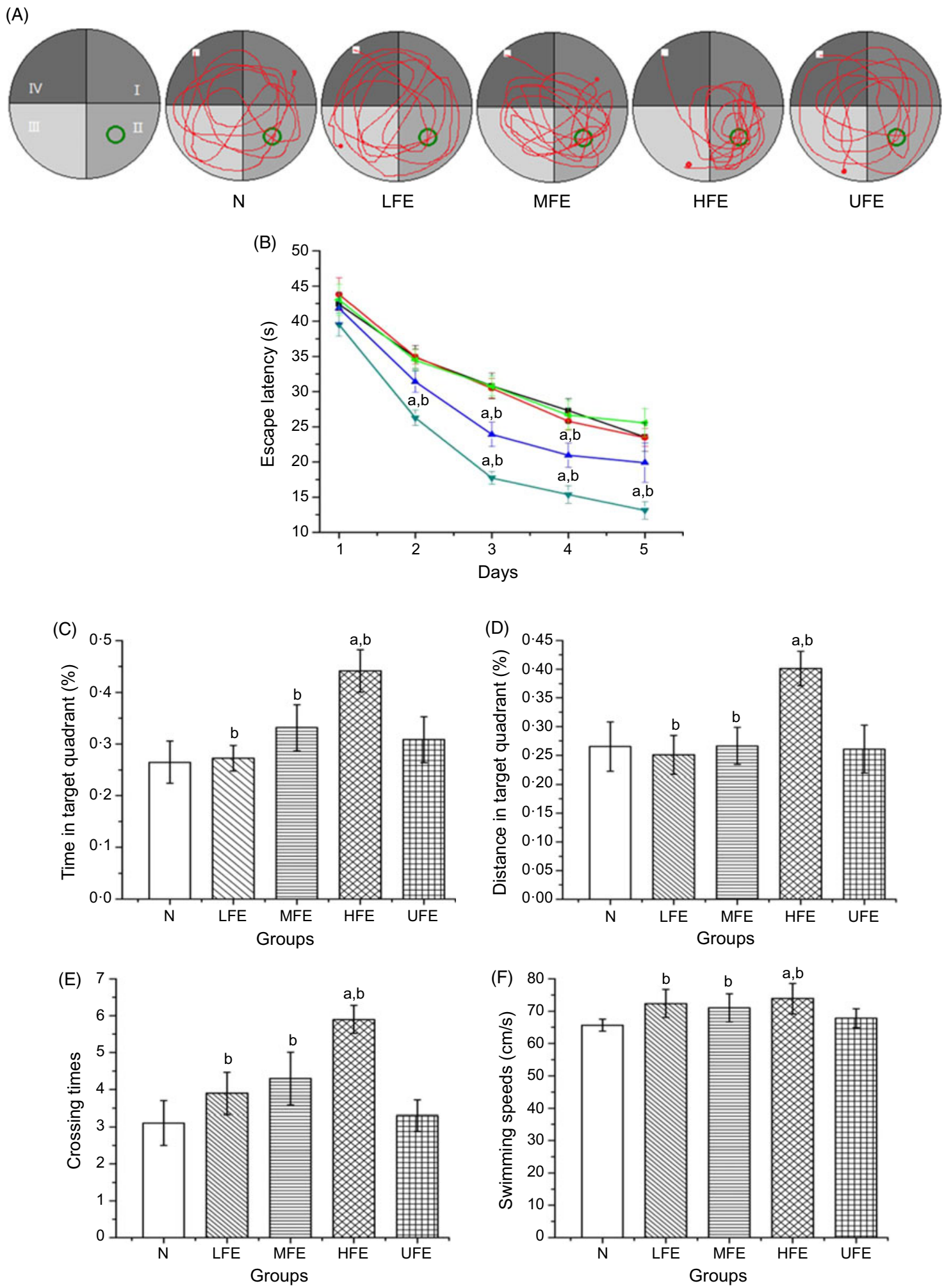

Fig. 2. (A) Swimming trajectory of mice from each group in probe trial; (B) escape latencies of each group in $5 \mathrm{~d}$ training trials; percentage of time (C) and distance (D) in target quadrant; number crossing over the position of the platform $(\mathrm{E})$ and swimming speeds $(\mathrm{F})$ in the probe trial. Values are presented as mean values with their standard errors. (B) $\longrightarrow-\mathrm{N} ; \multimap$, LFE; —-, MFE; $\longrightarrow$, HFE; - , UFE. a $P<0.05$ v. N group; ${ }^{b} P<0.05$ v. UFE group. N, normal group; LFE, low-dose of fermented peanut meal extract (FE) group; MFE, middle-dose of FE group; HFE, high-dose of FE group; UFE, unfermented extraction group. 
HFE group showed a larger percentage of swimming time and distance in target quadrant when compared with the $\mathrm{N}$ group and UFE group $(P<0.05)$. Furthermore, it can be found that the HFE group was crossed over the location where the hidden platform was located more frequently than the N group and UFE group (both $P<0.05$ ). There were no remarkable differences of the swimming speeds within different groups during the probe test $(P>0.05)$. It can be inferred from the results that the high dose of contraction from fermented PM can improve the learning and memory skills in mice.

At the same time, the platform was removed and the swimming trajectories of mice in each group were measured. It can be observed from Fig. 2(C) and (D) that the percentage of swimming time and distance in target quadrant in the HFE group were higher than that in N group and UFE group $(P<0 \cdot 05)$. Moreover, as seen in Fig. 2(E) and (F), the frequency of crossing the latent platform position in the HFE group was higher than that in $\mathrm{N}$ group and UFE group $(P<0.05)$. However, there were no significant differences in swimming speed among the groups in the probe test $(P>0.05)$. It can be inferred that PM after fermentation can improve the learning and memory ability of mice based on the experimental data.

\section{Changes of gut microbiota}

Fig. 3 displays the changes in the composition of GM at the phylum and genus levels in mice. It can be seen that the dominant gates are Bacteroidetes, Firmicutes and Proteobacteria. For Bacteroidetes, the relative content of group $\mathrm{B}$ was the highest (79.87\%), followed by group C (65.90\%), group A $(58.22 \%)$ and group D (45.10\%). At the level of door, the order of groups from high to low was group D (47.85\%), group A (29.28\%), group C (15.79\%) and group B (1.78\%). As can be seen in Fig. 3(A), the higher relative content was group B (17.73\%) and group C (17.60\%), followed by group A (11.63\%) and group D (6.92\%). As shown in Fig. 3(B) and Table 3, at the

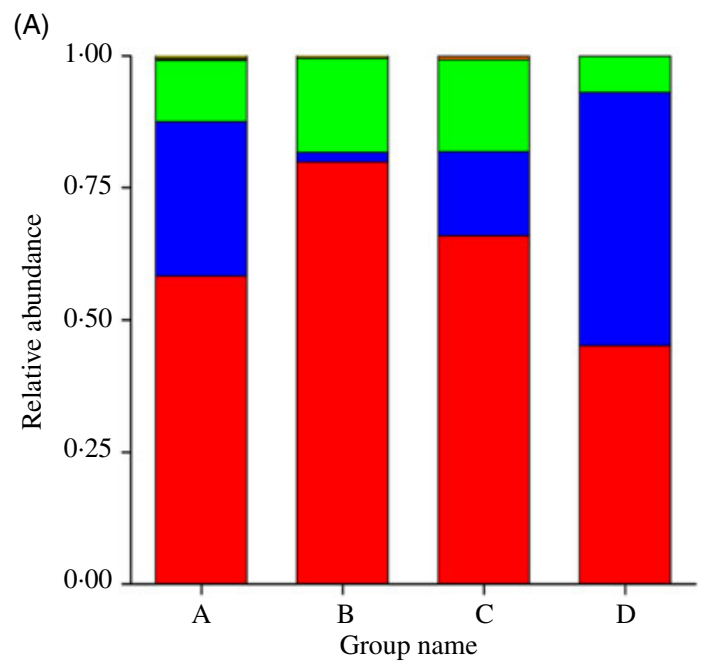

(B)

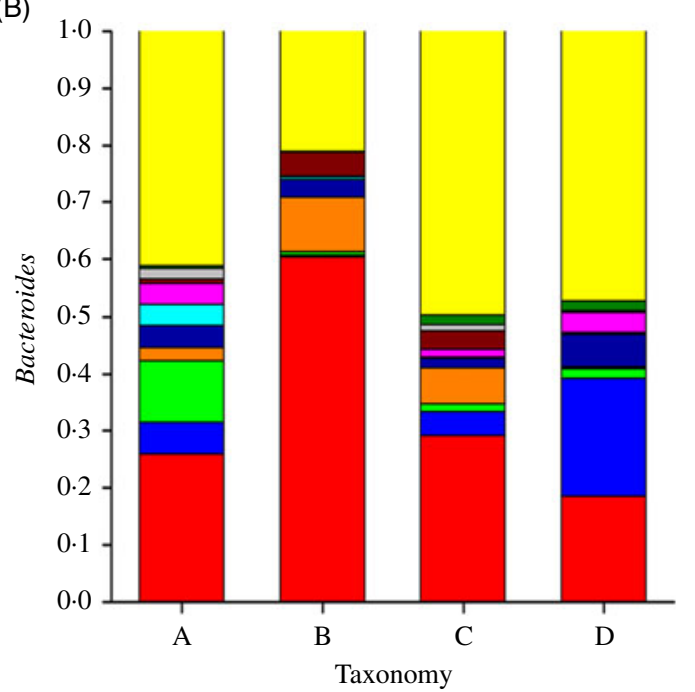

(C)

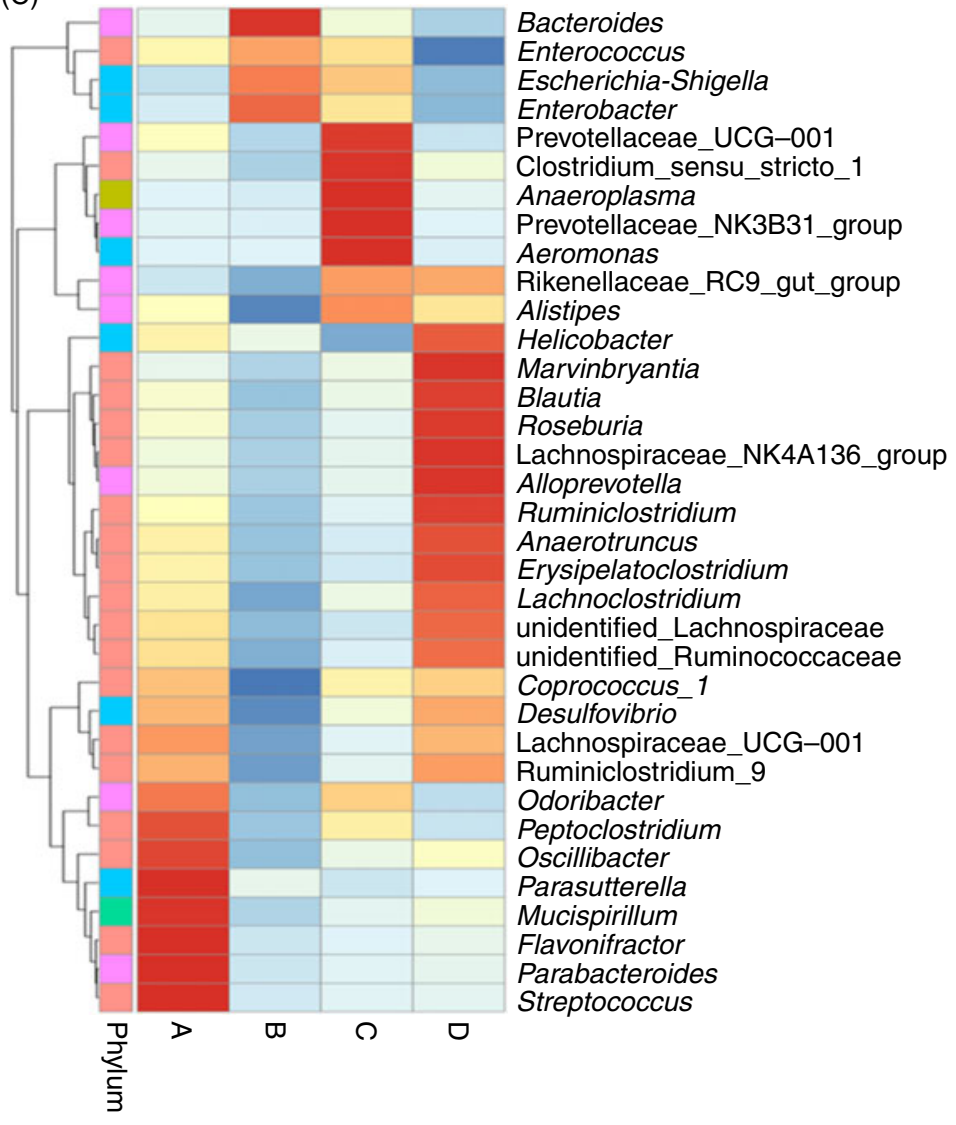

Fig. 3. (A) Relative abundances of the gut microbiota at the phylum level in mice of each group. The stack column diagram indicates the bacterial phyla composition of different groups, and different colours represent different kinds of bacterial phyla. (B) Relative abundances of the gut microbiota at the genus level in mice of each group. The stack column diagram indicates the bacterial genus composition of different groups, and different colours represent different kinds of bacterial genus. (C) Cluster map of species abundance. A, normal group; B, model group; C, natural recovery group; D, fermented peanut meal extract group. (A) $\square$, Others; $\square$, Cyanobacteria; $\square$, Actinobacteria; $\square$, Deferribacteres; $\square$, Tenericutes; $\square$, Proteobacteria; $\square$, Firmicutes; $\square$, Bacteroidetes. (B) $\square$, Others; $\square$, Rikenellaceae_RC9_gut_group; $\square$, Peptoclostridium; $\square$, Escherichia-Shigella; $\square$, Lachnospiraceae_UCG-001; $\square$, Parasutterella; $\square$, Helicobacter, $\square$, Enterobacter, $\square$, Parabacteroides; $\square$, Lachnospiraceae_NK4A136_group; $\square$, Bacteroides. (C) —, 1; , 0.5; , 0; , -0.5; , 0; , -1; Phylum:

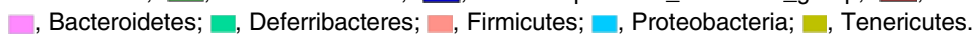


Table 3. Relative abundances of the gut microbiota at the genus level in mice of each group (\%)

\begin{tabular}{lrrrr}
\hline Taxonomy & \multicolumn{1}{c}{ A } & \multicolumn{1}{c}{ B } & \multicolumn{1}{c}{ C } & \multicolumn{1}{c}{ D } \\
\hline Bacteroides & 25.96 & 60.39 & 29.12 & 18.39 \\
Lachnospiraceae_NK4A136_group & 5.39 & 0.28 & 4.11 & 20.65 \\
Parabacteroides & 10.88 & 0.71 & 1.49 & 1.76 \\
Enterobacter & 2.27 & 9.46 & 6.23 & 0.32 \\
Helicobacter & 3.91 & 3.02 & 1.70 & 5.65 \\
Parasutterella & 3.59 & 0.54 & 0.18 & 0.33 \\
Lachnospiraceae_UCG-001 & 3.75 & 0.11 & 1.36 & 3.48 \\
Escherichia-Shigella & 0.80 & 4.16 & 3.34 & 0.15 \\
Peptoclostridium & 1.74 & 0.06 & 0.96 & 0.28 \\
Rikenellaceae_RC9_gut_group & 0.50 & 0.10 & 1.75 & 1.71 \\
Others & 41.20 & 21.16 & 49.75 & 47.28 \\
\hline
\end{tabular}

generic level, Bacteroides, Parabacteroides, Lachnospiraceae and Helicobacter were the most abundant bacteria in group A; Bacteroides, Enterobacter, Escherichia-Shigella were the main bacteria in group B; Bacteroides, Enterobacter, Lachnospiraceae and Escherichia-Shigella were the main bacteria in group C. Group D consisted mainly of Lachnospiraceae, Bacteroides and Helicobacter.

$\alpha$-Diversity was applied to analyse the complexity of the species diversity for the samples, including community richness (Chao1) and diversity indices (Shannon). The Chao1 value is often used to estimate the total number of species in a sample, while Shannon indicates the $\alpha$-diversity of intestinal microflora. The higher the Shannon is, the more evenly the distribution of microflora species is ${ }^{(22)}$. As depicted in Fig. 4, the species abundance of group B significantly decreased compared with that of group A $(P<0.05)$. The species abundance of groups $\mathrm{C}$ and D both notably increased compared with that of group $\mathrm{B}(P<0.05)$, and the Shannon value of group $\mathrm{D}$ was higher than that of group $\mathrm{C}(P<0 \cdot 05) . \alpha$-Diversities were further tested by comparing the $\alpha$-diversity indexes within the groups, and the results are shown in Table 4

The effect of FE on the species richness of intestinal bacteria in mice is shown in Fig. 5. There were 322 OTU in group A, 224 OTU in group $\mathrm{B}, 299$ OTU in group $\mathrm{C}$ and 323 OTU in group D, which indicates that FE can improve the richness of GM in antibiotic-induced diarrhoea mice. Moreover, as seen in Fig. 5(A), the dilution curve of each group was flat, suggesting that the sequencing quantity of the test can cover most of the micro-organisms in each sample. In addition, the curve width of group D was larger and smoother than that of group B,
Table 4. The $P$ values of the five main $\alpha$-diversity indices between groups

\begin{tabular}{lcccccc}
\hline$\alpha$-Index & A-B & A-C & A-D & B-C & B-D & C-D \\
\hline $\begin{array}{l}\text { Observed } \\
\text { species }\end{array}$ & $0.0231^{*}$ & 0.6529 & 0.6529 & $0.0478^{*}$ & $0.0114^{*}$ & 0.1775 \\
Shannon & $0.0320^{*}$ & 0.8826 & 0.5588 & $0.0406^{*}$ & $0.0126^{*}$ & $0.0467^{*}$ \\
Chao1 & $0.0240^{*}$ & 0.6558 & 0.6558 & $0.0493^{*}$ & $0.0119^{*}$ & 0.3816 \\
ACE & $0.0240^{*}$ & 0.6558 & 0.6558 & $0.0493^{*}$ & $0.0119^{*}$ & 0.3816 \\
PD-whole- & $0.0011^{\star *}$ & 0.1483 & $0.0316^{*}$ & $0.0094^{* *}$ & $0.0432^{*}$ & 0.3466 \\
tree & & & & & & \\
\end{tabular}

ACE, abundance-based coverage estimator; PD, phylogenetic diversity.

${ }^{*} P<0.05,{ }^{* *} P<0.01$

indicating that the species diversity of group $\mathrm{D}$ was more than that of group B. As shown in Fig. 5(B), compared with group A, the trend of group D was closer to that of group A, reflecting that the species diversity of fermented products was better than that of non-fermented products. It can be inferred that supplementation of natto fermentation products from PM can better alleviate and restore the richness of GM in mice.

\section{Discussion}

Growth performance is an important indicator of animal health and digestive function, while BMI and organ index are important manifestations of growth. Recently, more and more evidence shows that probiotic supplementation can promote animal growth and inhibit pathogenic micro-organisms ${ }^{(55)}$. In the present study, we found that the growth and development of mice supplemented with FE were better than that of mice supplemented with UFE, which may be related to the increase of nutritional characteristics of PM after fermentation. It was found that the protein content, polypeptide, polyphenol, DM content, organic acid and its digestibility in vitro of fermentation products were significantly increased. It is important to maintain a balance in essential amino acid content ${ }^{(35)}$. The increase of nutrients may be beneficial to the production of superoxide dismutase, which can eliminate the metabolic process of organisms, known as the first-line defensive role ${ }^{(56)}$. More importantly, antioxidants play an important role in enhancing learning and memory ability ${ }^{(57)}$, which is in accordance with the previous research ${ }^{(58)}$.

In addition, improving cognitive ability plays an important role in preventing some neurological diseases. There are many facts affecting the development of intelligence, while food
(A)

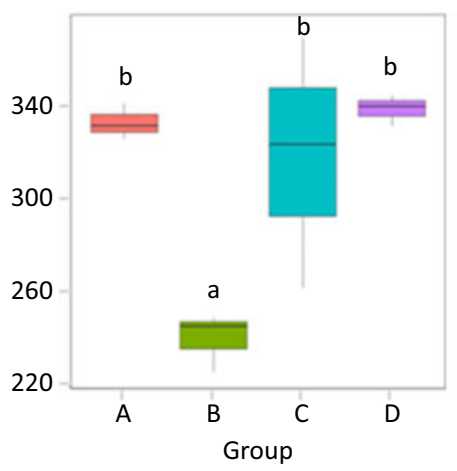

(B)

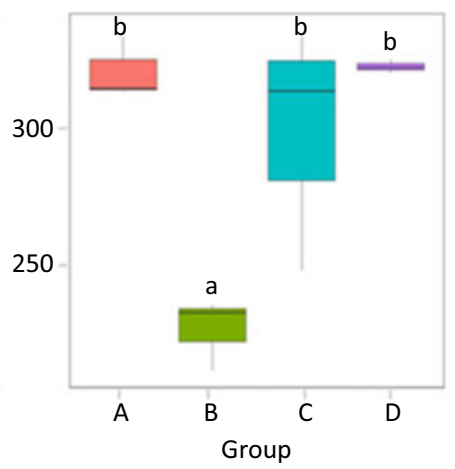

(C)

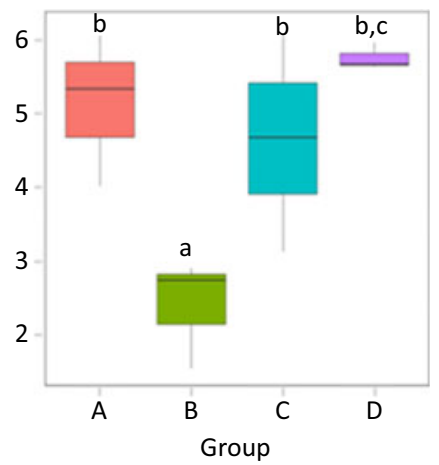

Fig. 4. (A) Chao1 values; (B) observed species and (C) Shannon values of the gut microbiota in mice of each group. Values are presented as box plots. A, normal group; $\mathrm{B}$, model group; $\mathrm{C}$, natural recovery group; $\mathrm{D}$, fermented peanut meal extract group. ${ }^{\mathrm{a}} P<0.05 \mathrm{v}$. group $\mathrm{A} ;{ }^{\mathrm{b}} P<0.05 \mathrm{v}$. group $\mathrm{B} ;{ }^{\mathrm{c}} P<0.05 \mathrm{v}$. group $\mathrm{C}$. 


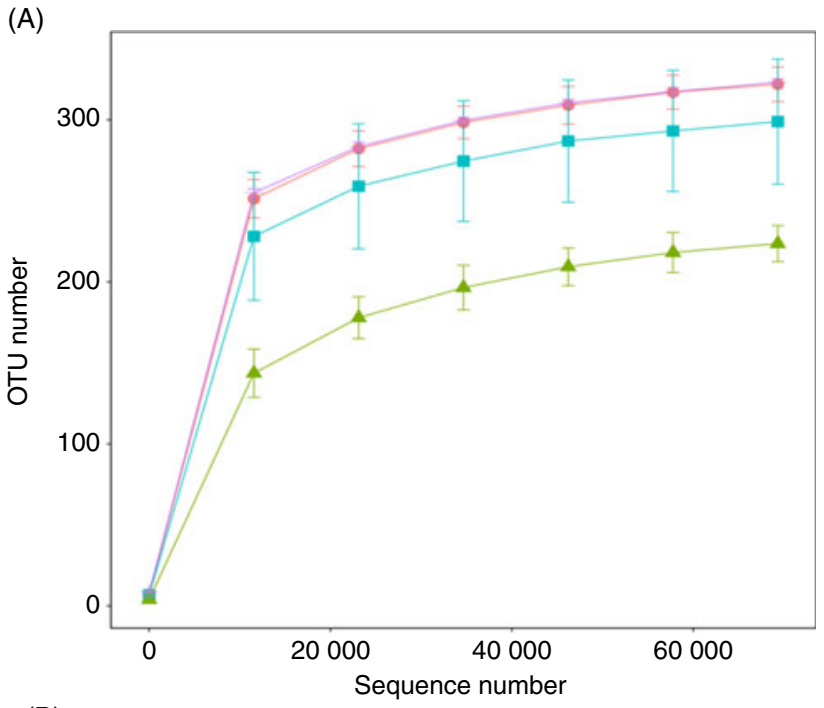

(B)

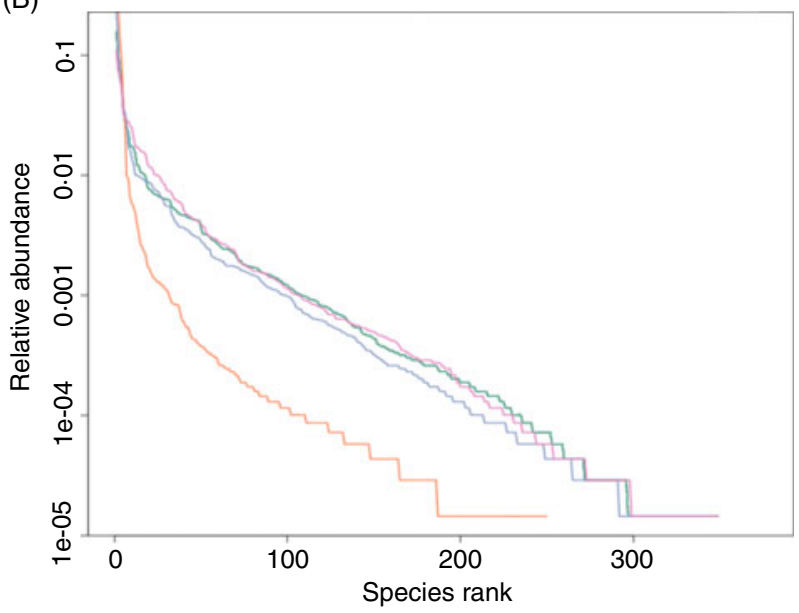

Fig. 5. (A) Rarefaction curves and (B) operational taxonomic unit (OTU) rank curves. A, normal group; B, model group; C, natural recovery group; D, fermented peanut meal extract group. (A) •, A; $\triangle, B$; $\mathbb{E}, \mathrm{C}$; +, D. (B) - A; $\longrightarrow, \mathrm{B} ;-, \mathrm{C} ;-, \mathrm{D}$.

supplement is certainly one of them. MWM testing is generally considered to be associated with hippocampal-dependent memory tasks ${ }^{(59)}$, which are often used to assess learning and memory skills in mice. Learning and memory include at least three original processes: acquisition, consolidation and retention $^{(60)}$. In the present study, training tests were designed to measure acquired and consolidated skills, while exploratory tests were designed to assess retention rates of learning and memory. It was found by us that the learning and memory abilities of mice in MFE group and HFE group were significantly improved, and the time ratio and distance ratio in target quadrant of mice in the HFE group were shorter. It can be seen from the side that the appropriate concentration of FE was conducive to improving learning and memory ability. This may be related to the improvement of nutrient composition of PM after fermentation and the increase of antioxidant enzymes activity in mice as well. Therefore, it can be inferred that supplementation of FE can improve the growth performance, learning and memory of mice, which is very important for promoting the growth and development of infants and adolescents and improving cognitive ability. In addition, further studies are needed to explore the possible underlying mechanism.

GM is a key determinant of nutritional intake, energy regulation and weight control. In addition to metabolic disorders, diet also plays a fundamental role in the formation of microflora ${ }^{(61)}$. Inappropriate utilisation of antibiotics can lead to the imbalance of GM. Dysbacteriosis, which means that the number and proportion of normal flora in the human body have changed, can lead to the imbalance of ecology ${ }^{(62)}$. It has been reported that different antibiotics have different effects on $\mathrm{GM}^{(63)}$, in which ampicillin shows moderate effects, and the higher the dose, the stronger the inhibitory effect. In the present study, each group of mice was given different extracts of PM for 1 month and then ampicillin was selected to induce diarrhoea in mice, resulting in GM imbalance, and the preventive effect of FE on flora imbalance was observed. The intestinal probiotics are mainly anaerobic bacteria, and the main pathogen is aerobic bacteria. B. natto is a subspecies of $B$. subtilis. It belongs to non-pathogenic bacteria in aerobic bacteria and secretes bacteriostatin-like substances. It can competitively inhibit the growth of aerobic bacteria and provide conditions for the growth of anaerobic bacteria, thus increasing the number of beneficial bacteria in intestine ${ }^{(64,65)}$. In order to investigate the specific changes of GM, we evaluated the relative abundance of dominant GM in mice by $16 \mathrm{~S}$ rDNA sequencing. The results showed that the number of Bacteroides in group B was higher at the level of phylum and genus. The main flora composition in group $\mathrm{C}$ was similar to that in group B. The number of Bacteroides in group $\mathrm{C}$ was lower than that in group $\mathrm{B}$, while still higher than that in groups $\mathrm{A}$ and $\mathrm{D}$. The number of Bacteroides in group D was lower. However, the number of Lachnospiraceae and Helicobacter was higher. It can be seen that FE can significantly reduce the number of Proteobacteria and Firmicutes species in the intestinal tract of experimental mice at the gate level, indicating that FE can improve antibiotic-induced diarrhoea and GM disorder, and achieve the role of regulating GM and improving diarrhoea. By $\alpha$ diversity analysis, $t$ test and Wilcoxon rank sum test, the diversity and species uniformity of group B were significantly lower than those of groups $\mathrm{C}$ and D. Shannon index analysis showed that species richness in group $\mathrm{D}$ was higher than that in other groups. The results reflected that FE can promote the growth of beneficial bacteria and inhibit the growth of harmful bacteria such as Bacteroides and Shigella. The results of highthroughput sequencing showed that FE can significantly improve the richness, diversity and uniformity of GM in antibiotic-induced diarrhoea mice. Our data are consistent with the results of reported studies, which have remarkable significance in preventing and even treating diseases ${ }^{(28)}$. In conclusion, FE can improve the overall health status by increasing the number of beneficial bacteria and improving GM, particularly those aspects related to the improvement and treatment of diarrhoea and intestinal diseases.

The brain-intestinal-microbial axis is a two-way 'communication system' which provides conditions for the interaction between 
gut microbes and brain, as well as brain and gut microbes. In addition, related studies have also shown that mental stress can activate the neural activity of the relevant parts of the central nervous system and, at the same time, pass signals through the brain-gut axis, change the gastrointestinal motility, activate intestinal mucosal immunity and destroy the intestinal mucosal barrier function ${ }^{(66)}$. From the experimental data, we can see that peanut powder fermented by natto bacteria can improve the GM of the experimental mice, promote the growth of beneficial bacteria and increase the richness and diversity of GM. MWM testing results showed that the average latency of FE mice was significantly shorter, with the significant increase of the number for cross-platforms, and the time of target quadrant exploration was higher than that of normal mice. It can be seen that the improvement of GM can promote the growth and development of mice and the improvement of cognitive ability, while a certain relationship exists between the two factors. Our study supports the growing scientific evidence that probiotic supplementation may have health and welfare benefits for the host ${ }^{(67)}$. Although the mechanism of the two factors still needs to be further explored, the experimental data may provide justification for future studies in the brain-intestinal-microbial axis and human clinical trials.

\section{Conclusion}

Due to amino acid imbalance and protein denaturation of PM, the efficient way to improve its utilisation rate remains challenging. In the present study, the fermentation of PM with $B$. natto was demonstrated to be beneficial to the improvement of growth performance, learning and memory ability in mice. It was also found that the fermented product can improve the function of GM imbalance and promote the growth of beneficial bacteria. Moreover, it can also inhibit the growth of harmful bacteria such as Bacteroides and Shigella and significantly improve the richness, diversity and uniformity of the GM. Our research provides a theoretical basis for further in-depth research on the theory of brain-gut axis and reflects a possible way to facilitate deep utilisation of PM.

\section{Acknowledgements}

The authors wish to thank Dr Tingting Zhang from the Biology Experimental Teaching Center of College of Life Sciences in Qingdao University for the use of some instruments and assistance with the measurements.

This work was financially supported by the Shandong Provincial Science and Technology Development Project (2014GSF120011) and Shandong Provincial Natural Science Foundation (ZR2019PH020).

Conception and design of the study: Y. H. and Z. L.; experiments: X. J., H. D. and Q. L.; collection of data: X. J., H. D. and Y. L.; analysis and interpretation of data: H. D., Q. L., Y. Z. and Y. W.; drafting of the manuscript: X. J. and H. D. and critical revision of the manuscript for important intellectual content: Y. W., A. M., Z. L. and Y. H.

The authors declare that there are no conflicts of interest.

\section{References}

1. Song DJ, Kang HY, Wang JQ, et al. (2014) Effect of feeding Bacillus subtilis natto on hindgut fermentation and microbiota of Holstein dairy cows. Asian-Australas J Anim Sci 27, 495-502.

2. Wu FC, Chang CW \& Shih IL (2013) Optimization of the production and characterization of milk clotting enzymes by Bacillus subtilis natto. Springerplus $\mathbf{2}, 33$.

3. Choo YM, Lee KS, Yoon HJ, et al. (2012) Antifibrinolytic role of a bee venom serine protease inhibitor that acts as a plasmin inhibitor. PLOS ONE 7, e32269.

4. Meng Y, Dong G, Zhang C, et al. (2016) Calcium regulates glutamate dehydrogenase and poly-gamma-glutamic acid synthesis in Bacillus natto. Biotechnol Lett 38, 673-679.

5. Jemil I, Mora L, Nasri R, et al. (2016) A peptidomic approach for the identification of antioxidant and ACE-inhibitory peptides in sardinelle protein hydrolysates fermented by Bacillus subtilis A26 and Bacillus amyloliquefaciens An6. Food Res Int 89, 347-358.

6. Chen ZF, Ai LY, Wang JL, et al. (2015) Probiotics Clostridium butyricum and Bacillus subtilis ameliorate intestinal tumorigenesis. Future Microbiol 10, 1433-1445.

7. Peng H, Wang JQ, Kang HY, et al. (2012) Effect of feeding Bacillus subtilis natto fermentation product on milk production and composition, blood metabolites and rumen fermentation in early lactation dairy cows. J Anim Physiol Anim Nutr 96, 506-512.

8. Li A, Wang Y, Li Z, et al. (2019) Probiotics isolated from yaks improves the growth performance, antioxidant activity, and cytokines related to immunity and inflammation in mice. Microb Cell Fact 18, 112.

9. Yamashita T, Oda E, Giddings JC, et al. (2003) The effect of dietary bacillus natto productive protein on in vivo endogenous thrombolysis. Pathophysiol Haemost Thromb 33, 138-143.

10. Brown JL \& Pollitt E (1996) Malnutrition, poverty and intellectual development. Sci Am 274, 38-43.

11. Terlabie NN, Sakyi-Dawson E \& Amoa-Awua WK (2006) The comparative ability of four isolates of Bacillus subtilis to ferment soybeans into dawadawa. Int J Food Microbio 106, $145-152$.

12. Chu J, Zhao H, Lu Z, et al. (2019) Improved physicochemical and functional properties of dietary fiber from millet bran fermented by Bacillus natto. Food Chem 294, 79-86.

13. Ming-Yu C, Ying-Ju C, Liang-Hung L, et al. (2019) Protective effects of hydrolyzed chicken extract (Probeptigen(R)/ Cmi-168) on memory retention and brain oxidative stress in senescence-accelerated mice. Nutrients 11, 1870.

14. Bickford PC, Gould T, Briederick L, et al. (2000) Antioxidantrich diets improve cerebellar physiology and motor learning in aged rats. Brain Res $\mathbf{8 6 6}, 211-217$.

15. Introini IB, McGaugh JL \& Baratti CM (1985) Pharmacological evidence of a central effect of naltrexone, morphine, and beta-endorphin and a peripheral effect of met- and leuenkephalin on retention of an inhibitory response in mice. Behav Neural Biol 44, 434-446.

16. Nishimura H, Higuchi O, Tateshita K, et al. (2006) Antioxidative activity and ameliorative effects of memory impairment of sulfur-containing compounds in Allium species. Biofactors 26, 135-146.

17. Chan YC, Hosoda K, Tsai CJ, et al. (2006) Favorable effects of tea on reducing the cognitive deficits and brain morphological changes in senescence-accelerated mice. J Nutr Sci Vitaminol 52, 266-273.

18. Subash S, Essa MM, Braidy N, et al. (2016) Consumption of fig fruits grown in Oman can improve memory, anxiety, and learning skills in a transgenic mice model of Alzheimer's disease. Nutr Neurosci 19, 475-483. 
19. Go J, Kim JE, Kwak MH, et al. (2016) Neuroprotective effects of fermented soybean products (Cheonggukjang) manufactured by mixed culture of Bacillus subtilis MC31 and Lactobacillus sakei 383 on trimethyltin-induced cognitive defects mice. Nutr Neurosci 19, 247-259.

20. Delzenne NM, Knudsen C, Beaumont M, et al. (2019) Contribution of the gut microbiota to the regulation of host metabolism and energy balance: a focus on the gut-liver axis. Proc Nutr Soc 78, 319-328.

21. Ma Y, Wang W, Zhang H, et al. (2018) Supplemental Bacillus subtilis DSM 32315 manipulates intestinal structure and microbial composition in broiler chickens. Sci Rep $\mathbf{8}, 15358$.

22. Jazi V, Boldaji F, Dastar B, et al. (2017) Effects of fermented cottonseed meal on the growth performance, gastrointestinal microflora population and small intestinal morphology in broiler chickens. Br Poult Sci 58, 402-408.

23. Quigley, EMM (2019) The spectrum of small intestinal bacterial overgrowth (SIBO). Curr Gastroenterol Rep 21, 3.

24. He Y, Mao C, Wen H, et al. (2017) Influence of ad libitum feeding of piglets with bacillus subtilis fermented liquid feed on gut flora, luminal contents and health. Sci Rep 7, 44553.

25. Hatanaka M, Yamamoto K, Suzuki N, et al. (2018) Effect of Bacillus subtilis C-3102 on loose stools in healthy volunteers. Benef Microbes $9,357-365$.

26. Yao KY, Zhang TZ, Wang HF, et al. (2018) Upgrading of byproduct from beverage industry through solid-state fermentation with Candida utilis and Bacillus subtilis. Lett Appl Microbiol 67, 557-563.

27. Li Y, Xu Q, Huang Z, et al. (2016) Effect of Bacillus subtilis CGMCC 1.1086 on the growth performance and intestinal microbiota of broilers. J Appl Microbiol 120, 195-204.

28. Zhang HL, Li WS, Xu DN, et al. (2016) Mucosa-reparing and microbiota-balancing therapeutic effect of Bacillus subtilis alleviates dextrate sulfate sodium-induced ulcerative colitis in mice. Exp Ther Med 12, 2554-2562.

29. Hayashi RM, Lourenço MC, Kraieski AL, et al. (2018) Effect of feeding Bacillus subtilis spores to broilers challenged with Salmonella enterica serovar Heidelberg Brazilian Strain UFPR1 on performance, immune response, and gut health. Front Vet Sci 5, 13

30. Chen Y, Kong Q, Chi C, et al. (2015) Biotransformation of aflatoxin B1 and aflatoxin G1 in peanut meal by anaerobic solid fermentation of Streptococcus thermophilus and Lactobacillus delbrueckii subsp. bulgaricus. Int J Food Microbiol 211, 1-5.

31. Shen N, Qin Y, Wang Q, et al. (2015) Production of succinic acid from sugarcane molasses supplemented with a mixture of corn steep liquor powder and peanut meal as nitrogen sources by Actinobacillus succinogenes. Lett Appl Microbiol 60, 544-551.

32. Yang X, Teng D, Wang X, et al. (2016) Enhancement of nutritional and antioxidant properties of peanut meal by biomodification with Bacillus licheniformis. Appl Biochem Biotechnol 180, 1227-1242.

33. Wang L, Zhao B, Li F, et al. (2011) Highly efficient production of D-lactate by Sporolactobacillus sp. CASD with simultaneous enzymatic hydrolysis of peanut meal. Appl Microbiol Biotechnol 89, 1009-1017.

34. Wang L, Niu Q, Hui Y, et al. (2015) Assessment of taste attributes of peanut meal enzymatic-hydrolysis hydrolysates using an electronic tongue. Sensors 15, 11169-11188.

35. Costa EF, Miller BR, Pesti GM, et al. (2001) Studies on feeding peanut meal as a protein source for broiler chickens. Poult Sci 80, 306-313.
36. Zhou G, Chen Y, Kong Q, et al. (2017) Detoxification of aflatoxin $\mathrm{B}(1)$ by Zygosaccharomyces rouxii with solid state fermentation in peanut meal. Toxins $\mathbf{9}, 42$.

37. Elumalai P, Park YJ, Cho M, et al. (2019) Red yeast rice fermentation with Bacillus subtilis B2 under blue light-emitting diodes increases antioxidant secondary products. Bioprocess Biosyst Eng 42, 529-539.

38. Garg R \& Thorat BN. (2014) Nattokinase purification by three phase partitioning and impact of t-butanol on freeze drying. Sep Purif Technol 131, 19-26.

39. Bunka F, Kriz O, Velickova A, et al. (2009) Effect of acid hydrolysis time on amino acid determination in casein and processed cheeses with different fat content. J Food Compos Anal 22, 224-232.

40. Gong R, Wang Y, Qi Y, et al. (2019) Effects of different processing methods on water soluble polysaccharide contents and monosaccharide compositions in Cervi Cornu Pantotrichum. Se Pu 32, 194-200.

41. Jonesa DL, Owena AG \& Farrarb JF (2002) Simple method to enable the high resolution determination of total free amino acids in soil solutions and soil extracts. Soil Biol Biochem 34, 1893-1902.

42. Zhu CZ, Zhang WG, Zhou GH, et al. (2013) Isolation and identification of antioxidant peptides from Jinhua ham. J Agric Food Chem 61, 1265-1271.

43. Fu W, Li Y, Yang X, et al. (2017) Antioxidant activity of flavonoids in Pinus yunnanensis needles. J Dali Univ 19, $247-259$.

44. Cuesta G, Suarez N, Bessio MI, et al. (2003) Quantitative determination of pneumococcal capsular polysaccharide serotype 14 using a modification of phenol-sulfuric acid method. J Microbiol Methods 52, 69-73.

45. Rhinehart-Jones TR, Fortier AH \& Elkins KL (1994) Transfer of immunity against lethal murine Francisella infection by specific antibody depends on host gamma interferon and T cells. Infect Immun 62, 3129-3137.

46. Tomas Pereira I \& Burwell RD (2015) Using the spatial learning index to evaluate performance on the water maze. Behav Neurosci 129, 533-539.

47. Ma L, Wang R, Dong W, et al. (2016) Long-term caloric restriction in mice may prevent age-related learning impairment via suppression of apoptosis. Behav Brain Res 315, 45-50.

48. Li XY (2016) Age- and brain region-specific changes of glucose metabolic disorder, learning, and memory dysfunction in early Alzheimer's disease assessed in APP/PS1 transgenic mice using (18)F-FDG-PET. Int J Mol Sci 17, 1707.

49. Kang J, Shin JW, Kim YR, et al. (2017) Nobiletin improves emotional and novelty recognition memory but not spatial referential memory. J Nat Med 71, 1-9.

50. Li S, Zhang C, Gu Y, et al. (2015) Lean rats gained more body weight than obese ones from a high-fibre diet. Br J Nutr 114 , 1188-1194.

51. Li S, Gao L, Chen L, et al. (2015) Continuously ingesting fructooligosaccharide can't maintain rats' gut Bifidobacterium at a high level. J Food Sci 80, M2530-M2534.

52. Magoc T \& Salzberg SL (2011) FLASH: fast length adjustment of short reads to improve genome assemblies. Bioinformatics 27, 2957-2963.

53. Caporaso JG (2010) QIIME allows analysis of high-throughput community sequencing data. Nat Methods 7, 335-336.

54. Wang Q, Garrity GM, Tiedje JM, et al. (2007) Naive Bayesian classifier for rapid assignment of rRNA sequences into the new bacterial taxonomy. Appl Environ Microbiol 73, 5261-5267. 
55. Liu L, Zeng D, Yang M, et al. (2019) Probiotic Clostridium butyricum improves the growth performance, immune function, and gut microbiota of weaning rex rabbits. Probiotics Antimicrob Proteins, 11, 1278-1292.

56. Steinmaus C, Ferreccio C, Yuan Y, et al. (2014) Elevated lung cancer in younger adults and low concentrations of arsenic in water. Am J Epidemiol 180, 1082-1087.

57. Xiang L, Sun K, Lu J, et al. (2011) Anti-aging effects of phloridzin, an apple polyphenol, on yeast via the SOD and Sir2 genes. Biosci Biotechnol Biochem 75, 854-858.

58. Zhang L, Bai K, Zhang J, et al. (2017) Dietary effects of Bacillus subtilis fmbj on the antioxidant capacity of broilers at an early age. Poult Sci 96, 3564-3573.

59. Tan Y, Nie S, Zhu W, et al. (2016) 7,8-Dihydroxyflavone ameliorates cognitive impairment by inhibiting expression of tau pathology in ApoE-knockout mice. Front Aging Neurosci 8, 287.

60. Yu J, Liu C, Zhang X, et al. (2005) Acupuncture improved cognitive impairment caused by multi-infarct dementia in rats. Physiol Behav 86, 434-441.
61. Yuan L, Li X, He S, et al. (2018) Effects of natural flavonoid Isoorientin on growth performance and gut microbiota of mice. J Agric Food Chem 66, 9777-9784.

62. Jernberg C, Lofmark S, Edlund C, et al. (2007) Long-term ecological impacts of antibiotic administration on the human intestinal microbiota. ISME J 1, 56-66.

63. Jernberg C, Lofmark S, Edlund C, et al. (2010) Long-term impacts of antibiotic exposure on the human intestinal microbiota. Microbiology 156, 3216-3223.

64. Beaugerie L \& Petit JC (2004) Antibiotic-associated diarrhoea. Best Pract Res Clin Gastroenterol 18, 337-352.

65. Khan TJ, Ahmed YM, Zamzami MA, et al. (2018) Effect of atorvastatin on the gut microbiota of high fat diet-induced hypercholesterolemic rats. Sci Rep $\mathbf{8}, 662$.

66. Kowalski K \& Mulak A (2019) Brain-gut-microbiota axis in Alzheimer's disease. J Neurogastroenterol Motil 25, 48-60.

67. Mazkour S, Shekarforoush SS \& Basiri S (2019) The effects of supplementation of Bacillus subtilis and Bacillus coagulans spores on the intestinal microflora and growth performance in rat. Iran J Microbiol 11, 260-266. 